\title{
CONCEPT FOR OPTICAL MARK PROCESSING
}

\author{
CIP, P[avel] \& HORAK, K[arel]
}

\begin{abstract}
This paper deals with processing and optical recognition of forms, questionnaires, mark papers or other filled paper types. Mark processing done by human is very time-consuming and after a long time, human brain and eyes are very tired. This leads to misinterpretation and errors. Optical recognition is more effective and faster. But first of all it is important to specify to the algorithms the structure of the evaluated form using basics components - preferably in XML code. This code says to the program, where the main parts of the forms are and which method is usable for its recognition. This paper describes proper ways for form designs and basic steps for detection of answers
\end{abstract}

Keywords: forms recognition, questionnaires processing, computer vision

\section{INTRODUCTION}

Processing of forms, answer sheets or papers with survey is very time consuming. Human can't focus on the stereotypic activity for a long time. Long term processing by single human leads to increasing error rates by eyestrain and fatigue of brain.

In some cases is necessary to evaluate large number of papers in a short time - for example: public opinion polls, marketing surveys, census, etc. Some companies use automatic systems for optical mark recognition. These systems must be smart, reliable, precise and faster than human. They are always based on methods of computer vision and image processing.

It should enable flawless processing of all specified (learned) form types, evaluate their values and create report about the results or compare answers with correct solutions.

\section{FORM CONCEPT}

Questionnaires with information for mass processing have the same design. To simplify the detection it is useful to define basic form sheet parts and inform the application which processing method should be used. In next section we recommend specific parts in forms.

\subsection{Calibration marks}

In automation recognition it is important to have form in right position. Stock of filled forms is putt into automation equipment which can get sheet separately and scanned into an image to the hard drive in PC. No one can guarantee that papers are scanned in the same angle of rotation or position. Therefore it is necessary to put some calibration marks on form's corners. In practice most useful shapes are crosses, rectangles, circles, triangles or lines which describes unique place in form.

\subsection{Header / Footer}

Header and footer included some information about faculty, company trademark and logo. It is possible to mention rules about exams here, time interval, sample of right answers' crossing, etc. This part is only information for user. If there is a form with more than one page, it is necessary to mark these pages by numbers (usually by number with its binary code for easier optical recognition).

\subsection{Personal data area}

This part is filled by personal data of user, student or interviewee. There are blocks for filling names, gender, actual date, name of subject, version of exam, etc. Some areas from this block could be recognized by OCR algorithms for easier data entries. OCR is the shortcut for Optical recognition of character mechanism in computer vision.

\subsection{Identification data area}

Identification part is similar to personal, but there are more blocks for optical recognition. The main reason for using this area is coherence to user. For example, in universities every student has own unique identification number. In final exam, it is very important to know this student's number for correct assignment with the university mark system. User, who filled this exam form, could write this UID number to the special block and it is recognized by OCR after.

If we can guarantee that this form will be received by the appropriate student, we can pre-print his UID here. There are lots of options for coding this number - for example in EAN barcode, QR code or another type of 2D code, or simple print in normal font useful for OCR.

\subsection{Answering data area}

This part is the most important on the form and has variable size opposed to the other. That size depends on number of questions. Answering part can be formed by table with columns and cells. Checkboxes for answers has shape of rectangle or circle. By crossing this part user chooses his answer. If he makes a mistake, it is able to fill this cell and put correct cross on right position. In this case it is necessary to attach the paper with questions. Another type of form could include questions directly in this answering area. There are questions with options of answers and interviewee (student or user) must cross the answer right here.

\subsection{Definition of form}

The formal form sheet description is stored in a special XML structure. For every form part it specifies its size, position, type and recognition methods for successful processing.

\section{STEPS OF RECOGNITION}

\subsection{Normalization to right position}

Every scanned form is transformed into grayscale image. After that three or four calibration symbols are localized. Methods for finding them are working on principle of transforming image into binary with labeling included objects. 
From knowledge of view calibration marks found there the best match or shape correlation with the same properties like extent, similarity with convex area or rotation. If we use four symbols in the corners, we must guarantee that one symbol is different from the others. It is useful for detection of rotation around $180^{\circ}$.

After finding these symbols, a form is transformed with projective or with simple affine matrix (in case of three symbols). We suppose that forms are scanned in automation machine sheet by sheet with pressure roll, so only rotation and position are deformation which may occur.

For affine/projective transformation, the value of the pixel located at $[\hat{x}, \hat{y}]^{T}$ in the output image, is determined by the value of the pixel located at $[\mathrm{x}, \mathrm{y}]^{\mathrm{T}}$ in the input image. The relationship between the input and the output point locations is defined by the equations in (Jan, 2006).

\subsection{Identification user}

In personal and identification part are filled important data. From XML description we know, what data we are finding, and which method we must use for recognition. The identifying of UID is possible by intermediate OCR technique (Cervinka, 2010).

We use extern methods and algorithms because some of them are protected by patent and their processing (decoding) is not part of this paper. These extern methods give us information in string or integer format for next processing.

\subsection{Finding answers}

Grayscale image of form is converted into binary by adaptive threshold. From definition of form design in XML, we know, where is the answer's area placed. There is a definition which type of answers is there too. We suppose two kinds of that - first is table with columns and cells for crossing, second are questions and answers together in sequence. In first case we take this part and count black pixels (in inverse binary image, white is the pixel of cells) in vertical projection with (1) on each column of the image line by line (Bishop, 2006; Pal, 1993).

$$
\operatorname{SUM}_{\text {vertical }}(x)=\sum_{y=1}^{H}\left\{\begin{array}{cc}
1 & \text { if im }(x, y)=1 \\
0 & \text { otherwise }
\end{array}\right.
$$

Where $\mathrm{im}$ is the image, $H$ is the height of image and $x$ is the number of actual column.

If the count of black pixels in a column is equal or similar to zero, the column with checkboxes is segmented. From knowledge that the space between groups of column is twice bigger than space between cells, we can segment this block of answers. After that we have three separately images. The same projection, but in horizontal direction, is used for these three columns for separating rows. At finally the method of vertical projection is used again for segmentation each checkbox (cell). Result of this projection is obvious on Fig.1.

In second case (form with questions and answers), previous steps are similar. From form design we know where answers (check boxes) are placed. Again by combination of vertical and horizontal projection we could find the answers. For recognition, if the box is marked by cross, filled or left blank, we use template matching with model of cross with using knowledge of percent filled area (Safraz, 2005).

Methods take into account that one question could have more than one right answer. This result is saved in structure with UID of student, user or with the other information from form for processing by next automatic system. This part is not point of view of this paper.

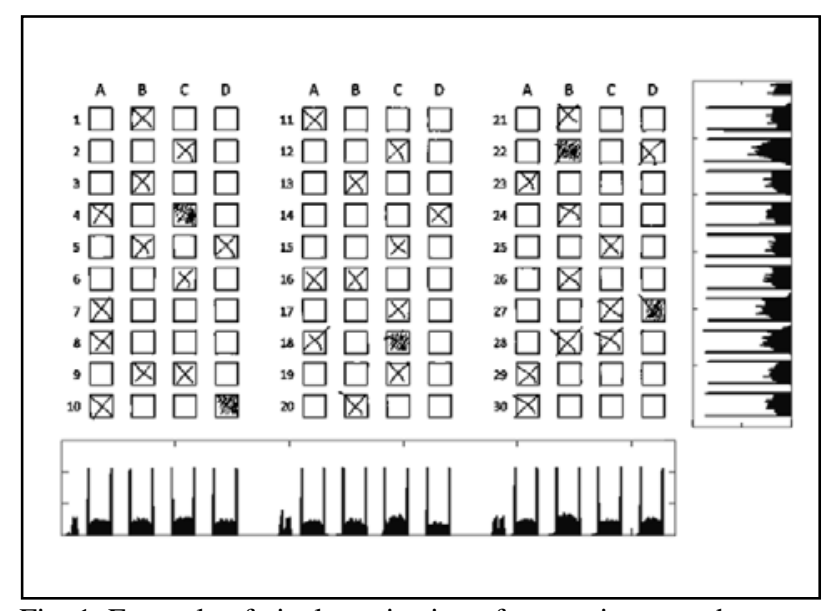

Fig. 1. Example of pixels projection of answering area; bottom: vertical projection; right: horizontal projection of last column

\section{CONCLUSION AND FUTURE WORK}

The proposed method of optical recognition mark processing was written in $\mathrm{C}++$ with using Computer Vision Library. Set of forms with 60 simple questions was filled by volunteers. They filled form with crossing of boxes. We tested both types of form (including variant with questions in form and sheet with more pages). Every form was scanned by automatic system and processed in PC (AMD, 1.8 GHz, 1GB RAM). Processing time was about 0.7 seconds with success more than $99.99 \%$. Errors were caused by user that not filled the wrong answer correctly or used a faint color pen. We defined some methods and principles for processing these forms. It is useful tool for evaluation large amounts of data. These methods are working with most popular type of forms. There is no problem to define basics' parts in unknown forms (by XML). In future work we would like to improve methods for recognition of user data in personal part by OCR methods, recognition of handwriting characters, etc.

\section{ACKNOWLEDGEMENTS}

This work has been supported by the Czech Ministry of Education under the project 1M0567 Centre for Applied Cybernetics and Grant Agency of the Czech Republic (102/09/H081 SYNERGY - Mobile Sensoric Systems and Network) and by Brno University of Technology.

\section{REFERENCES}

Bishop M. C. (2006). Pattern Recognition and Machine Learning, Springer-Verlag New York, Inc., Secaucus, NJ, USA

Cervinka, L.; Cip, P. \& Horak, K. (2010). Character Classifications by Radon Transform, Annals of DAAAM for 2010 \& Proceedings of the 21st International DAAAM Symposium, 20-23rd October 2010, Zadar, Croatia, ISSN 1726-9679, ISBN 978-3-901509-73-5, Katalinic, B. (Ed.), pp. 1399-1400, Published by DAAAM International Vienna, Vienna

Jan J. (2006). Medical Image Processing Reconstruction and Restoration: Concepts and Methods. Boca Raton: Francis \& Taylor, ISBN 0-8247-5849-8

Pal, N. (1993). A review on image segmentation techniques, Journal of Pattern Recognition, Vol. 26, p. 1277 - 1294

Safraz, M. (2005). Computer-Aided Intelligent Recognition Techniques and Applications, John Wiley \& Sons, Ltd. 519 p. ISBN 978-0-470-09414-3 